Supporting Information

\title{
Palladium-Catalyzed Enantioselective Ring-Opening of Oxabicyclic Alkenes with Organozinc Halides
}

\author{
Ming Li ${ }^{\mathrm{a}}$, Xiao Xia Yan, ${ }^{\mathrm{a}}$ Wei Hong ${ }^{\mathrm{b}}$, Xia-Zhen Zhu ${ }^{\mathrm{a}}$, Bo-Xun Cao ${ }^{\mathrm{a}}$, Jie Sun ${ }^{\mathrm{a}}$ and Xue-Long Hou ${ }^{\mathrm{a}, \mathrm{b}}$ * \\ ${ }^{a}$ State Key Laboratory of Organometallic Chemistry \\ ${ }^{b}$ Shanghai-Hong Kong Joint Laboratory in Chemical Synthesis, \\ Shanghai Institute of Organic Chemistry, Chinese Academy of Sciences
}

\section{General Methods}

All reactions were performed under a dry argon atmosphere. $\mathrm{Et}_{2} \mathrm{O}$ and THF were freshly distilled from sodium. Dichloromethane was freshly distilled from calcium hydride. Benzyl bromides were used as received without further purification. $\mathrm{CH}_{3} \mathrm{I}$ and $\mathrm{C}_{2} \mathrm{H}_{5} \mathrm{I}$ were redistilled before use. Benzylzinc bromides, ${ }^{1} \mathrm{CH}_{3} \mathrm{ZnI},{ }^{2}$ and $\mathrm{C}_{2} \mathrm{H}_{5} \mathrm{ZnI},{ }^{3} \mathrm{Pd}(\mathrm{dppb}) \mathrm{Cl}_{2},{ }^{4}$ and $\mathrm{Pd}\left(\mathrm{CH}_{3} \mathrm{CN}\right)_{2} \mathrm{Cl}_{2},{ }^{5}$ were prepared according to the literature procedures. Melting points were uncorrected. Enantiomeric excess were determined by HPLC using chiral column.

General procedure for palladium-catalyzed ring-opening of oxabicyclic alkenes with organozinc halides.

A solution of bis(acetonitrile)palladium dichloride (5 mol\%), $(S)-\operatorname{Pr}^{i}-\operatorname{PHOX} 6$ (5 mol\%), in dichloromethane $(1 \mathrm{~mL})$ was stirred at room temperature for two hours. To this solution was added at $10^{\circ} \mathrm{C}$ via canula a solution of oxabenzonorbornadiene $(1 \mathrm{mmol})$ in dichloromethane $(15$ $\mathrm{mL})$, followed by a solution of organozinc halide $(1.5 \mathrm{mmol})$ in THF. The resulting mixture was allowed to stir at $10^{\circ} \mathrm{C}$ until completion (monitored by TLC). Drops of water were added and the solution was stirred for half an hour, filtered through a short plug of celite. The solution was concentrated and the crude mixture was purified by flash chromatography on silica gel (ethyl acetate : petroleum $=1: 7$ ) to afford dihydronaphthol 3 . The ee value was determined by HPLC using chiral AD or OD column.

\section{(+)-(1S, 2S)-2-Benzyl-1,2-dihydronaphth-1-ol (3aa) ${ }^{6}$}

Yield $64 \% \cdot[\alpha]_{\mathrm{D}}^{20}=+159^{\circ}(c=0.59$, EtOH). 90\% ee (Chiralcel AD column , $n$-hexane $/ i$ $\left.\operatorname{PrOH}=80 / 20, \mathrm{t}_{\mathrm{R}}: 10.0 \mathrm{~min}, 12.4 \mathrm{~min}\right) .{ }^{1} \mathrm{H}$ NMR $\left(300 \mathrm{MHz}, \mathrm{CDCl}_{3}\right): \delta 1.62(\mathrm{~s}, 1 \mathrm{H}), 2.77-$ 
2.81(m, 1H), 2.89(dd, $J=8.0 \mathrm{~Hz}, 12.8 \mathrm{~Hz}, 1 \mathrm{H}), 3.15(\mathrm{dd}, J=7.7 \mathrm{~Hz}, 13.0 \mathrm{~Hz}, 1 \mathrm{H}), 4.47(\mathrm{dd}, J=$ $4.1 \mathrm{~Hz}, 7.0 \mathrm{~Hz}, 1 \mathrm{H}), 5.80(\mathrm{dd}, J=2.5 \mathrm{~Hz}, 9.6 \mathrm{~Hz}, 1 \mathrm{H}), 6.57(\mathrm{dd}, J=2.4 \mathrm{~Hz}, 9.6 \mathrm{~Hz}, 1 \mathrm{H}), 7.12-$ 7.25(m, 3H), 7.26-7.37 (m, 6H). MS (EI) m/z (rel) $236\left(\mathrm{M}^{+}, 1\right), 218$ (47), 145 (100), 144 (46), 127 (51), 115 (34), 91 (43).

\section{(+)-(1S, 2S)-2-(4-Bromo-benzyl)-1,2-dihydronaphth-1-ol (3ab)}

Yield $70 \%$. M.p. $115-117^{\circ} \mathrm{C} .[\alpha]_{\mathrm{D}}{ }^{20}=+182^{\circ}(c=0.43$, EtOH). $96 \%$ ee (Chiralcel OD column , $n$-hexane $/ i$ - PrOH $\left.=85 / 15, \mathrm{t}_{\mathrm{R}}: 13.1 \mathrm{~min}, 14.7 \mathrm{~min}\right) .{ }^{1} \mathrm{H} \mathrm{NMR}\left(300 \mathrm{MHz}, \mathrm{CDCl}_{3}\right): \delta$ 2.72-2.78 (m, 1H), $2.83(\mathrm{dd}, J=7.8 \mathrm{~Hz}, 13.0 \mathrm{~Hz}, 1 \mathrm{H}), 3.10(\mathrm{dd}, J=8.4 \mathrm{~Hz}, 12.8 \mathrm{~Hz}, 1 \mathrm{H}), 4.42(\mathrm{~d}$, $J=3.3 \mathrm{~Hz}, 1 \mathrm{H}), 5.75(\mathrm{dd}, J=2.4 \mathrm{~Hz}, 9.5 \mathrm{~Hz}, 1 \mathrm{H}), 6.57(\mathrm{dd}, J=2.5 \mathrm{~Hz}, 9.5 \mathrm{~Hz}, 1 \mathrm{H}), 7.11-7.32(\mathrm{~m}$, 6H), $7.39-7.46(\mathrm{~m}, 2 \mathrm{H}) .{ }^{13} \mathrm{C} \mathrm{NMR}\left(75 \mathrm{MHz}, \mathrm{CDCl}_{3}\right): \delta 139.0,136.2,132.4,131.4,131.0,129.8$, 128.7, 127.7, 127.6, 127.3, 126.6, 120.0, 69.3, 42.3, 34.7. MS (EI) m/z (rel) 314 (M+, 2), 296 (7), 145 (100), 144 (16), 127 (31), 115 (19), 91 (7). IR (KBr): 3345, 3201, 2951, 1487, 1335, $1011 \mathrm{~cm}^{-1}$. HRMS $\left(\mathrm{M}^{+}-\mathrm{H}_{2} \mathrm{O}\right)$ calcd. for $\mathrm{C}_{17} \mathrm{H}_{13} \mathrm{Br}$ : 296.02006; Found: 296.02013.

(+)-(1S, 2S)-2-(4'-Fluoro-benzyl)-1,2-dihydronaphth-1-ol (3ac)

Yield $57 \%$. M.p. $77-78^{\circ} \mathrm{C} .[\alpha]_{\mathrm{D}}{ }^{20}=+172^{\circ}(c=0.47, \mathrm{EtOH}) .90 \%$ ee (Chiralcel OD column, $n$-hexane $/ i$ - $\left.\mathrm{PrOH}=95 / 5, \mathrm{t}_{\mathrm{R}}: 21.4 \mathrm{~min}, 24.0 \mathrm{~min}\right) .{ }^{1} \mathrm{H} \mathrm{NMR}\left(300 \mathrm{MHz}, \mathrm{CDCl}_{3}\right): \delta 1.66(\mathrm{~d}, J=$ $6.9 \mathrm{~Hz}, 1 \mathrm{H}), 2.68-2.76(\mathrm{~m}, 1 \mathrm{H}), 2.82(\mathrm{dd}, J=7.8 \mathrm{~Hz}, 13.0 \mathrm{~Hz}, 1 \mathrm{H}), 3.10(\mathrm{dd}, J=8.6 \mathrm{~Hz}, 13.2 \mathrm{~Hz}$, $1 \mathrm{H}), 4.41(\mathrm{t}, J=5.2 \mathrm{~Hz}, 1 \mathrm{H}), 5.75(\mathrm{dd}, J=2.4 \mathrm{~Hz}, 9.6 \mathrm{~Hz}, 1 \mathrm{H}), 6.55(\mathrm{dd}, J=2.4 \mathrm{~Hz}, 9.6 \mathrm{~Hz}, 1 \mathrm{H})$, 6.95-7.10 (m, 2H), 7.13-7.30 (m, 6H). $\left.{ }^{13} \mathrm{C} \mathrm{NMR} \mathrm{(75MHz,} \mathrm{CDCl}_{3}\right): \delta 161.4\left(\mathrm{~d}, J_{C-F}=242.5 \mathrm{~Hz}\right)$, $136.3,135.6\left(\mathrm{~d}, J_{C-F}=3.5 \mathrm{~Hz}\right), 132.5,130.7,130.5,130.0,128.2\left(\mathrm{~d}, J_{C-F}=70.9 \mathrm{~Hz}\right), 127.6,126.9$ $\left(\mathrm{d}, J_{C-F}=45.8 \mathrm{~Hz}\right), 115.3,115.0,69.3,42.5\left(\mathrm{~d}, J_{C-F}=1.1 \mathrm{~Hz}\right), 34.5 . \quad \mathrm{MS}(\mathrm{EI}) \mathrm{m} / \mathrm{z}(\mathrm{rel}) 254\left(\mathrm{M}^{+}\right.$, 7), 255 (1), 236 (7), 145 (100), 127 (49), 115 (29), 91 (9). IR (KBr): 3370, 3296, 2930, 1599, 1508, 1454, 1335, 1220, 1155, 1080 $\mathrm{cm}^{-1}$. Anal. Calcd. for $\mathrm{C}_{17} \mathrm{H}_{15} \mathrm{FO}: \mathrm{C}, 80.29$; H, 5.95, Found: C, 80.33; H, 6.07.

(+)-(1S, 2S)-2-(4'-Methyl-benzyl)-1,2-dihydronaphth-1-ol (3ad)

Yield $65 \% .[\alpha]_{\mathrm{D}}^{20}=+21.3^{\circ}\left(c=0.80, \mathrm{CHCl}_{3}\right) .87 \%$ ee (Chiralcel AD column, $n$-hexane / $\left.i-\mathrm{PrOH}=80 / 20, \mathrm{t}_{\mathrm{R}}: 27.8 \mathrm{~min}, 35.7 \mathrm{~min}\right) .{ }^{1} \mathrm{H} \mathrm{NMR}\left(300 \mathrm{MHz}, \mathrm{CDCl}_{3}\right): \delta 2.35$ (s, 3H), 2.74-2.81 $(\mathrm{m}, 1 \mathrm{H}), 2.85(\mathrm{dd}, J=8.0 \mathrm{~Hz}, 12.6 \mathrm{~Hz}, 1 \mathrm{H}), 3.11(\mathrm{dd}, J=8.0 \mathrm{~Hz}, 12.8 \mathrm{~Hz}, 1 \mathrm{H}), 4.47(\mathrm{~d}, J=$ $3.6 \mathrm{~Hz}, 1 \mathrm{H}), 5.80(\mathrm{dd}, J=2.6 \mathrm{~Hz}, 9.3 \mathrm{~Hz}, 1 \mathrm{H}), 6.56(\mathrm{dd}, J=2.5 \mathrm{~Hz}, 9.6 \mathrm{~Hz}, 1 \mathrm{H}), 7.12-7.25$ (m, $6 \mathrm{H}), 7.28-7.43(\mathrm{~m}, 2 \mathrm{H}) .{ }^{13} \mathrm{C} \mathrm{NMR}\left(75 \mathrm{MHz}, \mathrm{CDCl}_{3}\right): \delta 136.9,136.4,135.5,132.6,130.4,129.1$, 
129.0, 128.6, 127.7, 127.6, 127.0, 126.5, 69.6, 42.5, 34.8, 21.0. MS (EI) m/z (rel) $250\left(\mathrm{M}^{+}, 1\right)$, 232 (49), 145 (100), 144 (74), 127 (65), 115 (65), 91 (24). IR (KBr): 3416, 2922, 1601, 1516, 1486, 1382, 1279, $1086 \mathrm{~cm}^{-1}$. HRMS $\left(\mathrm{M}^{+}-\mathrm{H}_{2} \mathrm{O}\right)$ calcd. for $\mathrm{C}_{18} \mathrm{H}_{16}: 232.12520$; Found: 232.12460 . (+)-(1S, 2S)-2-(3'-Methoxyl-benzyl)-1,2-dihydronaphth-1-ol (3ae)

Yield $44 \% .[\alpha]_{\mathrm{D}}^{20}=+66^{\circ}(c=1.02, \mathrm{EtOH}) .76 \%$ ee (Chiralcel AD column, $n$-hexane $/ i$ $\left.\mathrm{PrOH}=85 / 15, \mathrm{t}_{\mathrm{R}}: 15.5 \mathrm{~min}, 20.7 \mathrm{~min}\right) .{ }^{1} \mathrm{H} \mathrm{NMR}\left(300 \mathrm{MHz}, \mathrm{CDCl}_{3}\right): \delta 2.75-2.87(\mathrm{~m}, 2 \mathrm{H}), 3.11$ $(\mathrm{dd}, J=7.0 \mathrm{~Hz}, 12.2 \mathrm{~Hz}, 1 \mathrm{H}$ ), 3.79 (s, 3H), 4.47 (d, $J=3.9 \mathrm{~Hz}, 1 \mathrm{H}), 5.79$ (dd, $J=2.3 \mathrm{~Hz}, 9.6 \mathrm{~Hz}$, $1 \mathrm{H}), 6.55$ (dd, $J=2.4 \mathrm{~Hz}, 9.2 \mathrm{~Hz}, 1 \mathrm{H}$ ), 6.76-6.90 (m, 3H), 7.12 (d, $J=7.5 \mathrm{~Hz}, 1 \mathrm{H}), 7.17-7.30$ (m, $4 \mathrm{H}) .{ }^{13} \mathrm{C}$ NMR $\left(75 \mathrm{MHz}, \mathrm{CDCl}_{3}\right): \delta 159.6,141.7,136.4,132.5,130.2,129.3,128.6,127.6,127.0$, 126.5, 121.6, 114.9, 111.4, 69.6, 55.1, 42.3, 35.3. MS (ESI) m/z (rel) 289.1(100), 266.1(36), 305.0(8). IR (KBr): 3368, 3296, 2945, 1600, 1593, 1488, 1266, 1238, 1162, $1039 \mathrm{~cm}^{-1}$. HRMS(ESI) calcd. for $\mathrm{C}_{18} \mathrm{H}_{18} \mathrm{O}_{2}: 289.1199009$; Found: 289.1185430 .

(+)-(1S, 2S)-2-(3',4'-Dichloro-benzyl)-1,2-dihydronaphth-1-ol (3af)

Yield 53\%. $[\alpha]_{\mathrm{D}}{ }^{20}=+112^{\circ}(c=1.52, \mathrm{EtOH}) .89 \%$ ee (Chiralcel OD column, $n$-hexane $/ i$ $\left.\mathrm{PrOH}=95 / 5, \mathrm{t}_{\mathrm{R}}: 29.4 \mathrm{~min}, 33.3 \mathrm{~min}\right) .{ }^{1} \mathrm{H} \mathrm{NMR}\left(300 \mathrm{MHz}, \mathrm{CDCl}_{3}\right): \delta 2.69-2.75(\mathrm{~m}, 1 \mathrm{H}), 2.80$ $(\mathrm{dd}, J=8.0 \mathrm{~Hz}, 13.0 \mathrm{~Hz}, 1 \mathrm{H}), 3.09(\mathrm{dd}, J=8.3 \mathrm{~Hz}, 13.0 \mathrm{~Hz}, 1 \mathrm{H}), 4.42(\mathrm{~d}, J=3.9 \mathrm{~Hz}, 1 \mathrm{H}), 5.73$ (dd, $J=1.4 \mathrm{~Hz}, 9.4 \mathrm{~Hz}, 1 \mathrm{H}$ ), 6.57 (dd, $J=2.4 \mathrm{~Hz}, 9.7 \mathrm{~Hz}, 1 \mathrm{H}$ ), 7.12-7.15 (m, 2H), 7.19-7.32 (m, $3 \mathrm{H})$, 7.36-7.48 (m, 3H). ${ }^{13} \mathrm{C} \mathrm{NMR}\left(75 \mathrm{MHz}, \mathrm{CDCl}_{3}\right): 140.4,136.1,132.3,131.1,130.5,130.2$, 130.0, 129.4, 128.8, 128.7, 127.8, 127.6, 127.5, 126.7, 69.2, 42.3, 34.5. MS (EI) m/z (rel) 304 $\left(\mathrm{M}^{+}, 2\right), 286$ (2), 145 (100), 127 (34), 115 (26), 91 (7). IR (KBr): 3394, 2926, 1594, 1560, 1473, 1396, 1260, 1131, 1031 $\mathrm{cm}^{-1}$. HRMS $\left(\mathrm{M}^{+}\right)$calcd. for $\mathrm{C}_{17} \mathrm{H}_{14} \mathrm{Cl}_{2} \mathrm{O}: 304.04217$; Found: 304.04250 . (+)-(1S, 2S)-2-(3'-Bromobenzyl)-1,2-dihydronaphth-1-ol (3ag)

Yield: $35 \% .[\alpha]_{\mathrm{D}}{ }^{20}=+147.7^{\circ}(\mathrm{c}=2.265, \mathrm{EtOH})$; 95\%ee (Chiral OD, Flow rate: $0.7 \mathrm{~mL} /$ min, $n$-hexane $/ i$-PrOH $\left.=85 / 15,254 \mathrm{~nm}, \mathrm{t}_{\mathrm{R}} 14.53 \mathrm{~min}, 15.79 \mathrm{~min}\right) .{ }^{1} \mathrm{HNMR}\left(300 \mathrm{MHz}, \mathrm{CDCl}_{3}\right)$ : $\delta 2.72-2.76(\mathrm{~m}, 2 \mathrm{H}), 2.81(\mathrm{dd}, J=7.8,13.2 \mathrm{~Hz}, 1 \mathrm{H}), 3.13(\mathrm{dd}, J=7.8,12.6 \mathrm{~Hz}, 1 \mathrm{H}), 4.44(\mathrm{~s}, 1 \mathrm{H})$, 5.72-5.76(m, $1 \mathrm{H}), 6.572(\mathrm{dd}, J=2.1,9.3 \mathrm{~Hz}, 1 \mathrm{H}), 7.11-7.45(\mathrm{~m}, 8 \mathrm{H}) ;{ }^{13} \mathrm{CNMR}\left(75 \mathrm{MHz}, \mathrm{CDCl}_{3}\right): \delta$ $34.947,42.307,69.328,122.436,126.600,127.298,127.595,127.738,127.959$, 128.658, 129.248, 129.714, 129.912, 132.196, 132.397, 136.226, 142.498; MS (EI) m/z (rel) 297(3.45), 296(9.90), 145(100.00), 127(42.52), 115(129.11), 91(11.70), 77(9.79), 63(9.78), 51(7.64); IR: $3411,2852,1595,1567,1474,1453,1021,998,887,771,706,670,512,445 \mathrm{~cm}^{-1}$; Anal. Calcd for $\mathrm{C}_{17} \mathrm{H}_{15} \mathrm{BrO}$ : C, 64.78; H, 4.80. Found: C, 64.77; H, 4.77; 
(+)-(1S, 2S)-2-(2'-Bromobenzyl)-1,2-dihydronaphth-1-ol (3ah)

Yield:76\%. $[\alpha]_{\mathrm{D}}^{20}=+96.4^{\circ}(\mathrm{c}=3.700, \mathrm{EtOH}) ; 93 \%$ ee $($ Chiral OD, Flow rate: $0.7 \mathrm{~mL} /$ min, $n$-hexane $/ i$-PrOH $\left.=85 / 15,254 \mathrm{~nm}, \mathrm{t}_{\mathrm{R}}=14.14 \mathrm{~min}, 15.52 \mathrm{~min}\right) .{ }^{1} \mathrm{HNMR}\left(300 \mathrm{MHz}, \mathrm{CDCl}_{3}\right)$ : $\delta 2.29-2.99(\mathrm{~m}, 2 \mathrm{H}), 3.03(\mathrm{dd}, J=7.5,12.6 \mathrm{~Hz}, 1 \mathrm{H}), 3.11-3.3-21(\mathrm{dd}, J=8.1,12.9 \mathrm{~Hz}, 1 \mathrm{H}), 4.44$ $4.98(\mathrm{~m}, 1 \mathrm{H}), 5.78-5.83(\mathrm{~m}, 1 \mathrm{H}), 6.57(\mathrm{dd}, J=2.1,9.3 \mathrm{~Hz}, 1 \mathrm{H}), 7.08-7.58(\mathrm{~m}, 8 \mathrm{H}) ;{ }^{13} \mathrm{CNMR}$ $\left(75 \mathrm{MHz}, \mathrm{CDCl}_{3}\right): \delta 35.680,40.400,69.628,124.869,126.618,127.223,127.309,127.712$, $127.724,127.994,128.674,129.891,131.943,132.482,132.950,136.310,139.257 ;$ MS (EI) m/z (rel) 316(0.15), 314(0.19), 145(100.00), 127(52.85), 115(66.98), 77(82.53), 63(82.15), 51(63.83), 39(61.93); IR (KBr): 3369, 3045, 2931, 1565, 1471, 1374, 1297, 1080, 1045, 1024, 786, 749, 667, 442 $\mathrm{cm}^{-1}$; Anal. Calcd for $\mathrm{C}_{17} \mathrm{H}_{15} \mathrm{BrO}$ : C, 64.78; H, 4.80. Found: C, 64.72; H, 4.85.

(+)-(1S, 2S)-2-(2'-Methoxy-benzyl)-1,2-dihydronaphth-1-ol (3ai)

Yield:49.4\%; $[\alpha]_{\mathrm{D}}^{20}=+56.6(\mathrm{c}=0.795, \mathrm{EtOH}) ; 94 \%$ ee $($ Chiral AD; Flow rate: $0.7 \mathrm{~mL} /$ min, $n$-hexane $/ i$-PrOH $\left.=85 / 15,254 \mathrm{~nm}, \mathrm{t}_{\mathrm{R}}=17.210 \mathrm{~min}, 21.127 \mathrm{~min}\right) .{ }^{1} \mathrm{HNMR}(300 \mathrm{MHz}$, $\left.\mathrm{CDCl}_{3}\right): \delta 2.39(\mathrm{~d}, J=5.4 \mathrm{~Hz}, 1 \mathrm{H}), 2.84-2.95(\mathrm{~m}, 2 \mathrm{H}), 3.11-3.18(\mathrm{~m}, 1 \mathrm{H}), 3.84(\mathrm{~s}, 3 \mathrm{H}), 4.39-4.44(\mathrm{~m}$, $1 \mathrm{H}), 5.82-5.86(\mathrm{~m}, 1 \mathrm{H}), 6.56(\mathrm{dd}, J=2.1,9.0 \mathrm{~Hz}, 1 \mathrm{H}), 7.08-7.21(\mathrm{~m}, 8 \mathrm{H}) ;{ }^{13} \mathrm{CNMR}(75 \mathrm{MHz}$, $\left.\mathrm{CDCl}_{3}\right): \delta$ 29.922, 41.339, 55.348, 69.420, 110.319, 120.719, 126.394, 126.915, 127.408, 127.572, 127.853, 127.976, 128.431, 130.811, 131.300, 132.871, 136.247, 157.347; MS (EI) m/z (rel) 266(8.27), 145(65.98), 127(53.33), 91(100.00), 77(22.24), 65(26.06), 51(15.13); IR (KBr): 3364, 3290, 2931, 2834, 1587, 1494, 1464, 1289, 1243, 1182, 1052, 1033, 790, 782, 751, 558, 499; Anal. Calcd for $\mathrm{C}_{18} \mathrm{H}_{18} \mathrm{O}_{2}$ : C, 81.17; H, 6.81; Found: C, 81.02; H, 6.85.

(+)-(1S, 2S)-2-(2'-Methyl-benzyl)-1,2-dihydronaphth-1-ol (3ai)

Yield:20\%); $[\alpha]_{\mathrm{D}}{ }^{20}=+159.1^{\circ}(\mathrm{c}=1.000, \mathrm{EtOH}) ; 88 \%$ ee $($ Chiral AD, Flow rate: $0.7 \mathrm{~mL} /$ $\min , n$-hexane $/ i$-PrOH $\left.=80 / 20,254 \mathrm{~nm}, \mathrm{t}_{\mathrm{R}} 6.11 \mathrm{~min}, 6.75 \mathrm{~min}\right) .{ }^{1} \mathrm{HNMR}\left(300 \mathrm{MHz}, \mathrm{CDCl}_{3}\right): \delta$ $2.35(\mathrm{~s}, 3 \mathrm{H}), 2.77-2.81(\mathrm{~m}, 1 \mathrm{H}), 2.89(\mathrm{dd}, J=8.1,13.2 \mathrm{~Hz}, 1 \mathrm{H}), 3.13(\mathrm{dd}, J=8.1,13.5 \mathrm{~Hz}, 1 \mathrm{H})$, $4.48(\mathrm{~s}, 1 \mathrm{H}), 5.79-5.89(\mathrm{~m}, 1 \mathrm{H}), 6.56(\mathrm{dd}, J=2.1,9.3 \mathrm{~Hz}, 1 \mathrm{H}), 7.12-7.30(\mathrm{~m}, 8 \mathrm{H}) ;{ }^{13} \mathrm{CNMR}(75 \mathrm{MHz}$, $\left.\mathrm{CDCl}_{3}\right): \delta 19.506,32.375,40.953,69.862,125.839,126.254,126.553,127.134,127.573,127.655$, 128.589, 130.068, 130.374, 130.400, 132.517, 136.530, 138.097; IR : 3370, 3023, 2927, 2864, 1603, 1485, 1454, 1333, 1298, 1080, 1044, 791, 769, 741, 694, 570 $\mathrm{cm}^{-1}$; MS (EI) m/z (rel) 250(0.25), 232(44.33), 145(100.00), 127(65.64), 115(45.39), 105(43.84), 77(34.66), 65(12.45), 51(17.25), 39(20.33); HRMS for $\left(\mathrm{M}^{+}-\mathrm{H}_{2} \mathrm{O}\right) \mathrm{C}_{18} \mathrm{H}_{16}$ : 232.12520; Found: 232.12124.

(+)-(1S, 2S)-2-(4'-Bromo-benzyl)-1,2-dihydro-5,8-dimethylnaphth-1-ol (3bb) 
Yield $76 \% .[\alpha]_{\mathrm{D}}^{20}=+61.2^{\circ}(c=3.28, \mathrm{EtOH}) .95 \%$ ee (Chiralcel OD column, $n$-hexane $/ i$ $\left.\mathrm{PrOH}=97 / 3, \mathrm{t}_{\mathrm{R}}: 18.6 \mathrm{~min}, 20.7 \mathrm{~min}\right) .{ }^{1} \mathrm{H} \mathrm{NMR}\left(300 \mathrm{MHz}, \mathrm{CDCl}_{3}\right): \delta 2.31$ (s, 3H), $2.32(\mathrm{~s}, 3 \mathrm{H})$, 2.62-2.71(m, 1H), 2.93(dd, $J=8.0 \mathrm{~Hz}, 13.4 \mathrm{~Hz}, 1 \mathrm{H}), 3.14(\mathrm{dd}, J=8.6 \mathrm{~Hz}, 13.5 \mathrm{~Hz}, 1 \mathrm{H}), 4.59(\mathrm{dd}$, $J=3.9 \mathrm{~Hz}, 6.9 \mathrm{~Hz}, 1 \mathrm{H}), 5.75(\mathrm{dt}, J=1.8 \mathrm{~Hz}, 9.5 \mathrm{~Hz}, 1 \mathrm{H}), 6.75(\mathrm{dd}, J=3.1 \mathrm{~Hz}, 9.8 \mathrm{~Hz}, 1 \mathrm{H}), 6.99$ $(\mathrm{dd}, J=7.8 \mathrm{~Hz}, 16.5 \mathrm{~Hz}, 2 \mathrm{H}), 7.18-7.24(\mathrm{~m}, 2 \mathrm{H}), 7.42-7.47(\mathrm{~m}, 2 \mathrm{H}) .{ }^{13} \mathrm{C} \mathrm{NMR}\left(75 \mathrm{MHz}, \mathrm{CDCl}_{3}\right)$ : $139.1,134.3,133.4,131.7,131.5,131.0,130.3$, 130.1, 129.7, 128.7, 124.6, 119.9, 65.3, 42.0, 35.4, 18.9, 18.3. MS (EI) m/z (rel) 342 (M+, 4), 343 (1), 344 (4), 324 (3), 174 (15), 173 (100), 158 (25), 91 (3). IR (KBr): 3440, 2949, 1591, 1574, 1488, 1380, 1259, 1165, 1097, 1012 $\mathrm{cm}^{-1}$. HRMS (M+) calcd. for $\mathrm{C}_{19} \mathrm{H}_{19} \mathrm{BrO}: 342.06192$; Found: 342.06367.

\section{(+)-(1S, 2S)-2-(4'-Bromo-benzyl)-1,2-dihydro-6,7-dimethylnaphth-1-ol (3cb)}

Yield $72 \% .[\alpha]_{\mathrm{D}}^{20}=+32.7^{\circ}\left(c=1.70, \mathrm{CHCl}_{3}\right) .90 \%$ ee (Chiralcel OD column, $n$-hexane $/ i$ $\left.\mathrm{PrOH}=97 / 3, \mathrm{t}_{\mathrm{R}}: 24.6 \mathrm{~min}, 29.4 \mathrm{~min}\right) .{ }^{1} \mathrm{H}$ NMR $\left(300 \mathrm{MHz}, \mathrm{CDCl}_{3}\right): \delta 2.23(\mathrm{~s}, 3 \mathrm{H}), 2.27(\mathrm{~s}, 3 \mathrm{H})$, 2.66-2.71(m, 1H), 2.79(dd, $J=7.5 \mathrm{~Hz}, 13.2 \mathrm{~Hz}, 1 \mathrm{H}), 3.07(\mathrm{dd}, J=8.9 \mathrm{~Hz}, 13.1 \mathrm{~Hz}, 1 \mathrm{H}), 4.32(\mathrm{~d}, J$ $=4.2 \mathrm{~Hz}, 1 \mathrm{H}), 5.66(\mathrm{dd}, J=1.3 \mathrm{~Hz}, 9.6 \mathrm{~Hz}, 1 \mathrm{H}), 6.50(\mathrm{dd}, J=2.6 \mathrm{~Hz}, 9.6 \mathrm{~Hz}, 1 \mathrm{H}), 6.91(\mathrm{~s}, 1 \mathrm{H})$, $7.02(\mathrm{~s}, 1 \mathrm{H}), 7.16(\mathrm{~d}, J=8.6 \mathrm{~Hz}, 2 \mathrm{H}), 7.42(\mathrm{~d}, J=8.4 \mathrm{~Hz}, 2 \mathrm{H}) .{ }^{13} \mathrm{C} \mathrm{NMR}\left(75 \mathrm{MHz}, \mathrm{CDCl}_{3}\right): 139.2$, 136.9, 136.1, 133.9, 131.4, 131.0, 130.1, 129.1, 128.7, 127.9, 127.1, 119.8, 69.0, 42.6, 34.9, 19.5, 19.4. MS (EI) m/z (rel) 342 (M+, 5), 343 (2), 344 (5), 324 (1), 174 (14), 173 (100), 158 (25), 145 (13), 128 (6). IR (KBr): 3383, 2918, 1591, 1558, 1488, 1455, 1330, 1287, 1073, 1012 $\mathrm{cm}^{-1}$. HRMS $\left(\mathrm{M}^{+}\right)$calcd. for $\mathrm{C}_{19} \mathrm{H}_{19} \mathrm{BrO}: 342.06192$; Found: 342.06122 .

\section{(+)-(1S, 2S)-2-(4'-Bromo-benzyl)-1,2-dihydro-6,7-dibromonaphth-1-ol (3db)}

Yield $52 \% .[\alpha]_{\mathrm{D}}^{20}=+73.3^{\circ}\left(c=2.60, \mathrm{CHCl}_{3}\right) .81 \%$ ee(Chiralcel AD column, $n$-hexane $/ i$ $\left.\mathrm{PrOH}=90 / 10, \mathrm{t}_{\mathrm{R}}: 15.3 \mathrm{~min}, 25.3 \mathrm{~min}\right) .{ }^{1} \mathrm{H} \mathrm{NMR}\left(300 \mathrm{MHz}, \mathrm{CDCl}_{3}\right): \delta 1.70(\mathrm{br}, 1 \mathrm{H}), 2.71-2.77(\mathrm{~m}$, 2H), $3.06(\mathrm{dd}, J=6.9 \mathrm{~Hz}, 11.4 \mathrm{~Hz}, 1 \mathrm{H}), 4.46(\mathrm{br}, 1 \mathrm{H}), 5.85(\mathrm{~d}, J=9.6 \mathrm{~Hz}, 1 \mathrm{H}), 6.46(\mathrm{~d}, J=9.9 \mathrm{~Hz}$, $1 \mathrm{H}), 7.14(\mathrm{~d}, J=8.1 \mathrm{~Hz}, 2 \mathrm{H}), 7.38(\mathrm{~s}, 1 \mathrm{H}), 7.44(\mathrm{~d}, J=8.1 \mathrm{~Hz}, 2 \mathrm{H}), 7.55(\mathrm{~s}, 1 \mathrm{H}) .{ }^{13} \mathrm{C}$ NMR $\left(75 \mathrm{MHz}, \mathrm{CDCl}_{3}\right): 138.4,136.8,133.1,132.2,132.0,131.5,131.1,130.9,125.4,124.4,123.0$, 120.1, 68.4, 42.0, 34.1. MS (EI) m/z (rel) $470\left(\mathrm{M}^{+}, 3\right), 472$ (7), 473 (2), 474 (7), 475 (2), 476 (2), 454 (4), 393 (2), 224 (96), 222 (100), 171 (28), 169 (30). IR (KBr): 3316, 3042, 2915, 1651, 1589, 1542, 1487, 1470, 1343, 1110, 1072, 1049, 1010 $\mathrm{cm}^{-1}$. HRMS $\left(\mathrm{M}^{+}\right)$calcd. for $\mathrm{C}_{17} \mathrm{H}_{13} \mathrm{Br}_{3} \mathrm{O}$ : 469.85165; Found: 469.85381.

(+)-(1S, 2S)-2-(4'-Bromo-benzyl)-1,2-dihydro-1,4-dimethylnaphth-1-ol (3eb) 
Yield $52 \% .[\alpha]_{\mathrm{D}}^{20}=+179^{\circ}(c=0.36$, EtOH). $64 \%$ ee( Chiralcel AD column, $n$-hexane $/ i$ $\left.\mathrm{PrOH}=97.5 / 2.5, \mathrm{t}_{\mathrm{k}}: 11.9 \mathrm{~min}, 21.3 \mathrm{~min}\right) .{ }^{1} \mathrm{H} \mathrm{NMR}\left(300 \mathrm{MHz}, \mathrm{CDCl}_{3}\right): \delta 1.53(\mathrm{~s}, 3 \mathrm{H}), 1.84(\mathrm{~s}$, $1 \mathrm{H}), 2.01(\mathrm{t}, J=1.2 \mathrm{~Hz}, 3 \mathrm{H}), 2.28(\mathrm{dd}, J=11.1 \mathrm{~Hz}, 12.5 \mathrm{~Hz}, 1 \mathrm{H}), 2.48-2.54(\mathrm{~m}, 1 \mathrm{H}), 3.03(\mathrm{dd}, J=$ $4.6 \mathrm{~Hz}, 12.5 \mathrm{~Hz}, 1 \mathrm{H}), 5.50(\mathrm{dd}, J=1.5 \mathrm{~Hz}, 5.7 \mathrm{~Hz}, 1 \mathrm{H}), 6.97-7.04(\mathrm{~m}, 2 \mathrm{H}), 7.22-7.37(\mathrm{~m}, 5 \mathrm{H})$, 7.58-7.61 (m, 1H). ${ }^{13} \mathrm{C}$ NMR (75MHz, $\left.\mathrm{CDCl}_{3}\right): 141.3,139.6,133.6,131.1,131.0,130.9,127.9$, 127.8, 127.3, 123.4, 123.3, 119.6, 74.4, 47.6, 34.3, 29.1, 19.1. MS (EI) m/z (rel) 342 (M+, 5), 344 (5), 343 (1), 174 (15), 173 (100), 158 (30), 145 (14), 128 (8), 91 (3). IR (KBr): 3465, 2970, 2922, 1643, 1592, 1488, 1363, 1072, $1012 \mathrm{~cm}^{-1}$. HRMS $\left(\mathrm{M}^{+}\right)$calcd. for $\mathrm{C}_{19} \mathrm{H}_{19} \mathrm{BrO}: 342.06192$; Found: 342.06026.

\section{(+)-2-Methyl-1,2-dihydronaphth-1-ol (10) ${ }^{7}$}

Yield $80 \% \cdot[\alpha]_{\mathrm{D}}^{20}=+91.1^{\circ}\left(c=1.21, \mathrm{CHCl}_{3}\right) .80 \%$ ee (Chiralcel AD column, $n$-hexane $/ i$ $\left.\mathrm{PrOH}=97.5 / 2.5, \mathrm{t}_{\mathrm{R}}: 19.0 \mathrm{~min}, 20.4 \mathrm{~min}\right) .{ }^{1} \mathrm{H} \mathrm{NMR}\left(300 \mathrm{MHz}, \mathrm{CDCl}_{3}\right): \delta 1.27(\mathrm{~d}, J=7.7 \mathrm{~Hz}, 3 \mathrm{H})$, $1.57(\mathrm{~d}, J=8.0 \mathrm{~Hz}, 1 \mathrm{H}), 2.62-2.68(\mathrm{~m}, 1 \mathrm{H}), 4.51(\mathrm{dd}, J=4.8 \mathrm{~Hz}, 7.4 \mathrm{~Hz}, 1 \mathrm{H}), 5.80(\mathrm{dd}, J=3.1 \mathrm{~Hz}$, $9.4 \mathrm{~Hz}, 1 \mathrm{H}), 6.53(\mathrm{dd}, J=2.5 \mathrm{~Hz}, 9.5 \mathrm{~Hz}, 1 \mathrm{H}), 7.10-7.14(\mathrm{~m}, 1 \mathrm{H}), 7.21-7.45(\mathrm{~m}, 3 \mathrm{H}) . \mathrm{MS}(\mathrm{EI})$ m/z (rel) 160 (M+, 64), 161 (8), 159 (13), 145 (79), 131 (100), 128 (30).

\section{(+)-2-Ethyl-1,2-dihydronaphth-1-ol (11) ${ }^{7}$}

Yield $81 \% .[\alpha]_{\mathrm{D}}^{20}=+47.6^{\circ}\left(c=1.21, \mathrm{CHCl}_{3}\right) .92 \%$ ee (Chiralcel OD column, $n$-hexane $/ i$ $\left.\mathrm{PrOH}=100 / 1.25, \mathrm{t}_{\mathrm{R}}: 25.9 \mathrm{~min}, 29.2 \mathrm{~min}\right) .{ }^{1} \mathrm{H} \mathrm{NMR}\left(300 \mathrm{MHz}, \mathrm{CDCl}_{3}\right): \delta 1.08(\mathrm{t}, J=7.5 \mathrm{~Hz}, 3 \mathrm{H})$, $1.56-1.68(\mathrm{~m}, 1 \mathrm{H}), 1.75-1.87(\mathrm{~m}, 1 \mathrm{H}), 2.22-2.39(\mathrm{~m}, 1 \mathrm{H}), 4.60(\mathrm{~d}, J=4.8 \mathrm{~Hz}, 1 \mathrm{H}), 5.83(\mathrm{dd}, J=$ $3.0 \mathrm{~Hz}, 9.3 \mathrm{~Hz}, 1 \mathrm{H}), 6.53(\mathrm{dd}, J=2.7 \mathrm{~Hz}, 9.9 \mathrm{~Hz}, 1 \mathrm{H}), 7.09-7.12(\mathrm{~m}, 1 \mathrm{H}), 7.17-7.33(\mathrm{~m}, 3 \mathrm{H}) . \mathrm{MS}$ (EI) m/z (rel) $174\left(\mathrm{M}^{+}, 8\right), 175$ (1), 157 (17), 145 (50), 144 (100), 128 (6), 116 (23). 


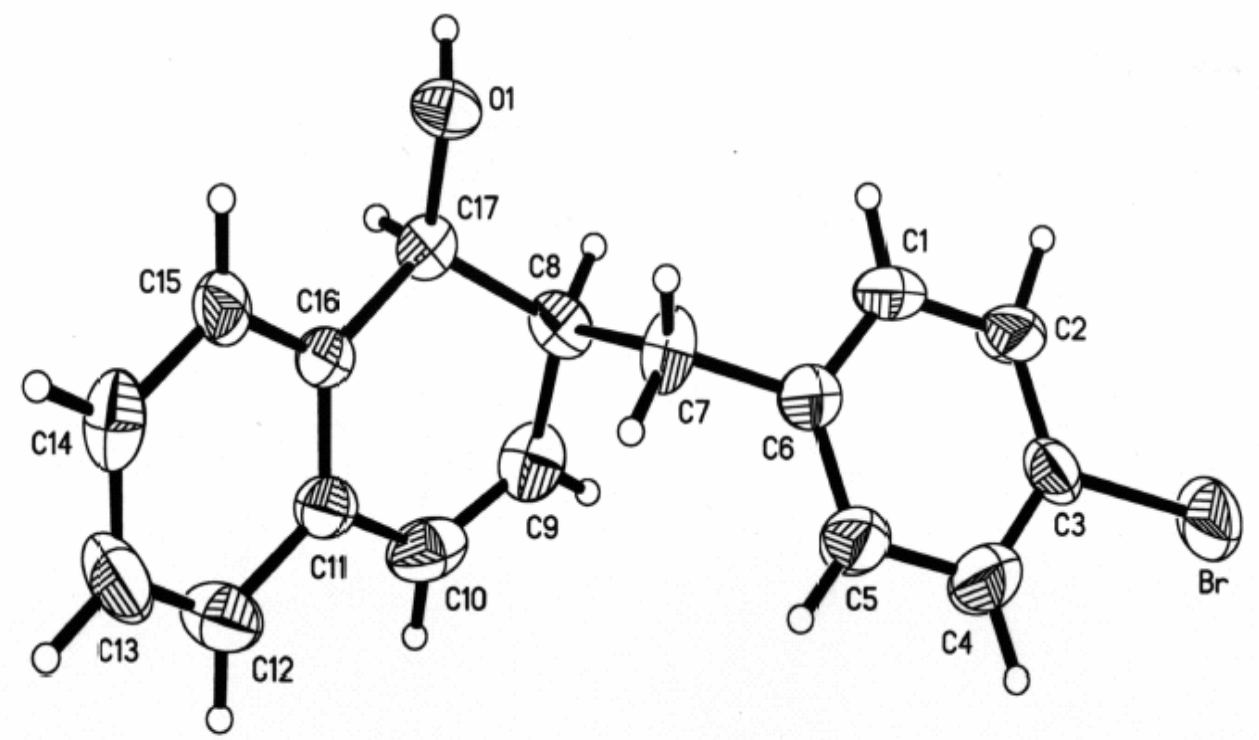

X-ray Structure of compound 3ab. Only one enantiomer was detected.

\section{References:}

1. (a) Berk. S. C.; Yeh, M. C. P.; Jeong, N.; Knochel, P. Orgnometallics 1990, 9, 30533064. (b) Knochel, P.; Yeh, M. C. P.; Berk, S. C.; Talbert, J. J. Org. Chem. 1988, 53, 2390.

2. Hermann, W. A. (ed) Synthetic Method of Organometallic and Inorganic Chemistry, Thieme, 1999, 5. 117.

3. Friedrich. E. C.; Jassawalla, J. D. C. J. Org. Chem. 1977, 44, 4224.

4. Makhaev, V. D.; Dzhabieva, Z. M.; Konovalikhin, S. V.; D'yachenko, O. A.; Belov, G. P. Russ. J. Coord. Chem. (Transl. of Koord. Khim.), 1996 22, 563-567.

5. Andrews, M. A.; Chang, T. C. T.; Cheng, C. W. F.; Emge, T. J.; Kelly, K. P.; Koetzle, T. F. J. Am. Chem. Soc., 1984, 106, 5913-5920.

6. Duan, J.-P.; Cheng, C.-H. Tetrahedron lett. 1993, 34, 4019-4022.

7. Lautens, M.; Renaud, J.-L.; Hiebert, S. J. Am. Chem. Soc. 2000, 122, 1804-1805. 
